\title{
Quantum Fields in Anti-de Sitter Space and the Maldacena Conjecture
}

\author{
Nelson R. F. Braga \\ Instituto de Física \\ Universidade Federal do Rio de Janeiro, RJ, 21945-970 - Brazil
}

Received on 7 February, 2002

\begin{abstract}
We review the relation between the Maldacena conjecture, also known as AdS/CFT correspondence, and the so-called holographic principle that seems to be an essential ingredient for a quantum gravity theory. We also illustrate the idea of holography by showing that the curvature of the anti-de Sitter space reduces the number of degrees of freedom making it possible to find a mapping between a quantum theory defined on the bulk and another defined on the corresponding boundary.
\end{abstract}

\section{Introduction}

The interest of theoretical physicists in studying quantum fields in anti de Sitter (AdS) space is not new[1]. In particular the question of the quantization of fields in this space circunventing the problem of the lack of a Cauchy surface was addressed in[2, 3]. There was, however, a remarkable increase in the attention devoted to this subject since the appearance of the Maldacena conjecture[4] (see $[5,6]$ for reviews) on the equivalence (or duality) of the large $N$ limit of $S U(N)$ superconformal field theories in $n$ dimensions and supergravity and string theory in anti de Sitter spacetime in $n+1$ dimensions also called AdS/CFT correspondence. This correspondence was elaborated by Gubser, Klebanov and Polyakov [7] and by Witten [8] interpreting the boundary values of bulk fields as sources of boundary theory correlators.

The large scientific impact caused by Maldacena work can be associated with (at least) two reasons:

- (i) It represents an explicit example of a holographic mapping between two quantum theories that live in different dimensions. That means: a realization of the Holographic Principle.

- (ii) It also represents an important step in the search for a string theory description of QCD that would lead to a formulation for QCD at low energies (where perturbative calculations can not be used because of the strong coupling)

As a remark let us mention that the large $\mathrm{N}$ limit of $\mathrm{SU}(\mathrm{N})$ gauge theories was studied by 't Hooft a long time ago. He considered an expansion in the parameter $1 / N$ were QCD gets a simpler form (planar digrams dominate $\rightarrow$ topological structure like strings) [9].

\section{Holographic principle}

The original motivation for the holographic principle was the study of black hole entropy. Let us start with the following question: What happens with the entropy of the Universe when some portion of matter (say a rocket or anything else) is absorbed by a black hole? Classicaly (we mean without quantum mechanics) black holes can only absorb particles. So, one could not associate any temperature with them. Thus it would make no sense to think that the black hole itself has some entropy and the entropy of the Universe would not be conserved when we throw some matter inside it.

Quantum effects however change this picture. The thermal radiation found out by Hawking lead himself and Bekenstein[10, 11] to develop a thermodynamical model for black holes. Based on the analysis of the dynamics of black holes they found a generalised form of the second law of thermodynamics where a black hole has an entropy proportional to area of its horizon

$$
S_{B H}=\frac{A}{4 \hbar G}
$$

The generalized form of the second law of thermodynamics reads:

$$
\Delta S_{B H}+\Delta S_{\text {Rest of Univ. }} \geq 0
$$

One should be able to find a way of calculating this entropy by summing up degrees of freedom of black 
holes. This is still an open problem because it would depend essentially on the formulation of a satisfactory quantum theory for gravity. There are however some important results in this direction for example calculating black hole entropy using string theory as refs. $[12,13]$.

An interesting consequence of eq. (1) is that it contrasts with our standard idea (without taking gravity into account) that the entropy is an extensive quantity. That means: entropy should be proportional to the volume not to the area. This result lead 't Hooft and Susskind to formulate the Holographic Principle:

"Physics of a quantum system with gravity in some volume $V$ can be described in terms of degrees of freedom that can be contained in its boundary".

The general idea is that some systems tend naturally to black holes and for the others we can find processes that transform them into black holes and increase their entropy. So: the maximum entropy is limited by the boundary area (in Planck units). That means: Quantum mechanics plus gravity in 3 spatial dimensions is equivalent to an image that can be mapped in a bidimensional projection. The area of the boundary in units of Planck area represents the maximum number of degrees of freedom in the interior volume. It is presently believed that a (candidate to ) quantum gravity theory should satisfy this principle.

\section{Implementation of Malda- cena conjecture}

Soon after Maldacena's seminal article, Gubser, Klebanov and Polyakov[7] and Witten[8] have elaborated the general conjecture showing how to calculate Physical quantities of a conformal theory in the boundary of an anti-de Sitter $(A d S)$ space in terms of a bulk theory. In order to see how this correspondence holds, let us first remind that an anti-de Sitter space of $n+1$ dimensions $\left(A d S_{n+1}\right)$ is a space of constant negative curvature that can be taken as a $\mathrm{Hy}-$ perboloid in a larger $n+2$ dimensional flat space with coordinates $\left(X^{0}, X^{1}, \ldots, X^{n}, X^{n+1}\right)$ and metric $\eta_{a b}=$ $\operatorname{diag}(+,-,-, \ldots,-,+)$ :

$$
\left(X_{0}\right)_{2}+\left(X_{n+1}\right)^{2}-\sum_{i=1}^{n}\left(X_{i}\right)^{2}=\Lambda^{2}=\text { constant },
$$

We can introduce coordinate systems inside $A d S$ like the global coordinates $\rho, \tau, \Omega_{i}$, more used before the discovery of the $A d S / C F T$ correspondence, defined by:

$$
X_{0}=\Lambda \sec \rho \cos \tau
$$

$$
\begin{aligned}
X_{i} & =\Lambda \tan \rho \Omega_{i} \\
X_{n+1} & =\Lambda \sec \rho \sin \tau
\end{aligned}
$$

where $\sum_{i=1}^{n} \Omega_{i}^{2}=1,0 \leq \rho<\pi / 2,0 \leq \tau<2 \pi$

Note that the time variable $\tau$ is compact, so we must "unwrap" it by actually considering the $A d S$ covering space (that means an infinite set of copies of $A d S$ spaces in the $\tau$ direction.

For the AdS/CFT correspondence the so called Poincaré coordinates $\left(z, x^{i}, t\right) \quad$ (with $z \geq 0$ ) are more usefull. They are defined by the relations

$$
\begin{aligned}
X_{0} & =\frac{1}{2 z}\left(z^{2}+\Lambda^{2}+\vec{x}^{2}-t^{2}\right) \\
X_{i} & =\frac{\Lambda x^{i}}{z} \\
X_{n} & =-\frac{1}{2 z}\left(z^{2}-\Lambda^{2}+\vec{x}^{2}-t^{2}\right) \\
X_{n+1} & =\frac{\Lambda t}{z}
\end{aligned}
$$

and the corresponding measure reads

$$
d s^{2}=\frac{\Lambda^{2}}{z^{2}}\left((d z)^{2}+(d \vec{x})^{2}-(d t)^{2}\right)
$$

The AdS boundary, where the Conformal Field Theory is defined corresponds to the region $z=0$ plus a point at infinity: $z=\infty$.

Witten[8] has interpreted the $A d S / C F T$ correspondence in terms of a holographyc mapping: boundary values of fields that have dynamics defined inside the AdS space act as sources of correlation functions of the boundary conformal field theory (CFT). The simplest illustrative example is that of a scalar massless field (massless) in $A d S$ (more details can be found in $[7,8,16,17]$. Taking a scalar field in the bulk, with boundary value

$$
\phi\left(z, x^{i}, t\right) \rightarrow \phi_{0}\left(x^{i}, t\right) \quad \text { as } z \rightarrow 0
$$

and conformal operators $\mathcal{O}\left(x^{i}, t\right)$ on the boundary, the generator of correlation functions for this CFT operators (now: $x^{i}, t \equiv x$ for simplicity)

$$
Z\left[\phi_{0}\right]=\left\langle\exp \int d^{n} x \phi_{0}(x) \mathcal{O}(x)\right\rangle
$$

such that

$$
\frac{\delta}{\delta \phi_{0}\left(x_{1}\right)} \frac{\delta}{\delta \phi_{0}\left(x_{2}\right)} Z\left[\phi_{0}\right]=\left\langle\mathcal{O}\left(x_{1}\right) \mathcal{O}\left(x_{2}\right)\right\rangle
$$


where the fields $\phi_{0}$ act as sources will be calculated from the on shell action $I(\phi)$ for the bulk scalar field $Z\left[\phi_{0}\right] \Longleftrightarrow \exp \{-I(\phi)\}$

Considering the action for a scalar field in curved spacetime

$$
L=\frac{1}{2} \int d^{n+1} x \sqrt{g} \partial_{\mu} \phi \partial^{\mu} \phi
$$

with equation of motion

$$
\nabla_{\mu} \nabla^{\mu} \phi=\frac{1}{\sqrt{g}} \partial_{\mu}\left(\sqrt{g} \partial^{\mu} \phi\right)=0 .
$$

we can relate the field in AdS bulk in terms of boundary values:

$$
\phi(z, x)=c \int d^{n} x^{\prime} \frac{(z)^{n}}{\left((z)^{2}+\left(x-x^{\prime}\right)^{2}\right)^{n}} \phi_{0}\left(x^{\prime}\right) .
$$

and write the on shell action as

$$
I[\phi]=-\frac{c n}{2} \int d^{n} x d^{n} x^{\prime} \frac{\phi_{0}\left(x^{\prime}\right) \phi_{0}(x)}{\left(x-x^{\prime}\right)^{2 n}}
$$

So we find, for example, the two point correlation function for the operators $\mathcal{O}(x)$

$$
\langle\mathcal{O}(x) \mathcal{O}(y)\rangle \sim \frac{1}{(x-y)^{2 n}}
$$

as expected from conformal invariance.

Let us remark that the operators $\mathcal{O}$ of the CFT are not in general scalar fields but rather composite operators whose conformal dimension depends on the dimension of the space.

\section{Counting degrees of freedom in $A d S$ space}

Considering the geometry of $A d S$ space in Poincare coordinates we see that a hypersurface of area $\Delta A$ corresponding to $\Delta x^{1} \ldots \Delta x^{n-1}$ at some small $z=\delta$ will generate a finite volume $\Delta V$ if we move it along $z$ till $z \rightarrow \infty$

$$
\begin{gathered}
\Delta A=\int \frac{d^{n-1} x}{(z / \Lambda)^{n-1}}=\left(\frac{\Lambda}{\delta}\right)^{n-1} \Delta x^{1} \ldots \Delta x^{n-1} \\
\Delta V=\int \frac{d^{n-1} x d z}{(z / \Lambda)^{n}}=\Lambda \frac{\Delta A}{n-1} .
\end{gathered}
$$

This fact is illustrated in the figure bellow.

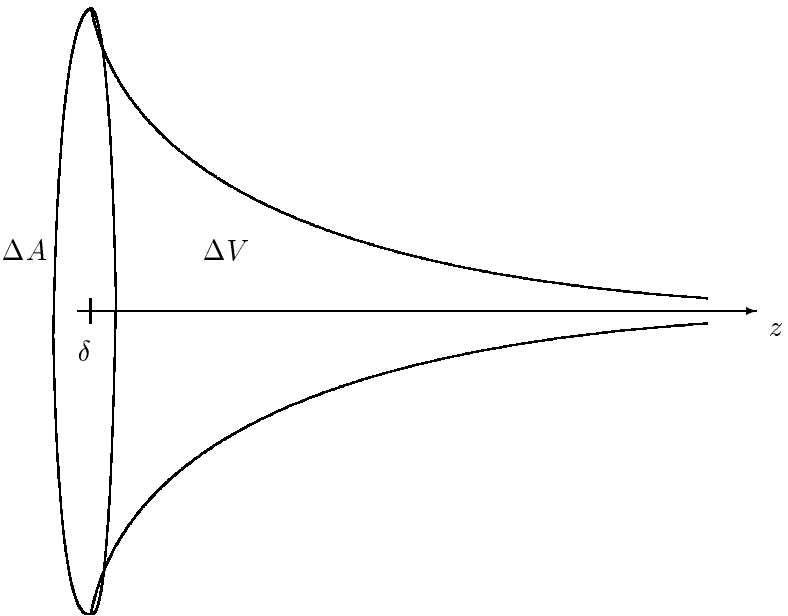

Figure 1.

If we associate (in a regularised way) degrees of freedom with volume cells of the space we see that we can map the $\Delta V$ cells in area cells of $\Delta A$. This reduction of degrees of freedom associated with the fact that the volume is proportional to the area should be reflected in a quantum theory in $A d S$. How can it be so? The hint comes from the analisys of the Cauchy problem in the $A d S$ space considered in [2,3]: massless particles can enter or left the $A d S$ from spatial infinity in finite times. So a consistent quantization (that means a well defined Cauchy problem) requires a compactification of the space. One has to add a surface at infinity where boundary conditions have to be imposed. (Like the cover of a box: nothing enters or lefts the space). These references considered global coordinates where the boundary is the hypersurface $\rho=\pi / 2$.

Now considering the Cauchy problem in Poincaré Coordinates, particles without mass may come from or go to $z \rightarrow \infty$ in a finite time. But how can we compactify the $\mathrm{z}$ coordinate that has infinite range? The answer $[18,19,20]$ is that one needs to introduce another coordinate chart $\left(z^{\prime}, x^{i}, t\right)$ related to the original one by

$$
\frac{1}{z^{\prime}}=\frac{1}{\delta}-\frac{1}{z}
$$

were we take the range of the coordinates $z$ as $\delta \leq z \leq$ $R$ in order to avoid the singularity at the origin and to stop the first chart at some arbitrarily large $R$. For $z^{\prime}$ we take and $\delta \leq z^{\prime} \leq R^{\prime}$ where $R^{\prime}=\delta R /(R-\delta)$ in order to map the rest of the space. The two sets must be compact because we need to impose boundary conditions that match them togheter.

Now the "point at infinity" in the $z$ coordinate is mapped at $z^{\prime}=\delta$

The important consequence of this representation of the compact $A d S$ space in terms of two compact coordinate charts is that even in the Poincare coordinate chart the field spectrum is discrete 


$$
\Phi(z, \vec{x}, t)==\sum_{p=1}^{\infty} \int \frac{d^{n-1} k}{(2 \pi)^{n-1}} \frac{z^{n / 2} J_{\nu}\left(u_{p} z\right)}{R w_{p}(\vec{k}) J_{\nu+1}\left(u_{p} R\right)}\left\{a_{p}(\vec{k}) e^{-i w_{p}(\vec{k}) t+i \vec{k} \cdot \vec{x}}+c . c .\right\}
$$

The important consequence of this discretization of the field spectrum is that it makes possible to find a one to one mapping between bulk and boundary theories. This would not be possible if the spectrum were continous. We can understand this by an analogy with the fact that it is possible to find a one to one mapping of an enumerable set of lines into one single line but it is not possible to map (one to one) a plane and a line. This is explained in [21] were an explicit mapping between scalar fields in $A d S$ space and scalar fields on the boundary is presented.

\section{References}

[1] C. Fronsdal, Physical Review D12, 3819 (1975).

[2] S. J. Avis, C. J. Isham, and D. Storey, Phys. Rev. D18, 3565 (1978).

[3] P. Breitenlohner and D. Z. Freedman, Phys. Lett. B115, 197 (1982); Ann. Phys. 144, 249 (1982).

[4] J. Maldacena, Adv. Theor. Math. Phys. 2, 231 (1998).

[5] O. Aharony, S.S. Gubser, J. Maldacena, H. Ooguri, and Y. Oz, Phys. Rept 323, 183 (2000).

[6] J. L. Petersen, Int. J. Mod. Phys. A14, 3597 (1999).

[7] S. S. Gubser , I.R. Klebanov, and A.M. Polyakov, Phys. Lett. B428, 105 (1998).
[8] E. Witten, Adv. Theor. Math. Phys. 2, 253 (1998).

[9] G. 't Hooft, Nucl. Phys. B72, 461 (1974).

[10] J. D. Bekenstein, Lett. Nuov. Cim. 4, 737 (1972), Phys. Rev. D7, 2333 (1973).

[11] S. W. Hawking, Phys. Rev. D13, 2460 (1976).

[12] A. Strominger and C. Vafa, Phys. Lett. B379, 99 (1996).

[13] J. M. Maldacena and A. Strominger, JHEP 9802, 014 (1998).

[14] G. 't Hooft, "Dimensional reduction in quantum gravity" in Salam Festschrifft, 1993, gr-qc/9310026.

[15] L. Susskind, J. Math. Phys. 36, 6377 (1995).

[16] D. Z. Freedman, S.D. Mathur, A. Matusis, and L. Rastelli, Nucl. Phys. B546, 96 (1999).

[17] W. Mueck and K. S. Viswanathan, Phys. Rev. D58, 041901 (1998).

[18] H. Boschi-Filho and N. R. F. Braga, Nucl. Phys. B608, 319 (2001).

[19] H. Boschi-Filho e N. R. F. Braga, Phys. Lett. B505, 263 (2001).

[20] H. Boschi-Filho and N. R. F. Braga, Phys. Rev. D66, 025005 (2002).

[21] H. Boschi-Filho and N. R. F. Braga, Phys. Lett. B525, 164 (2002). 